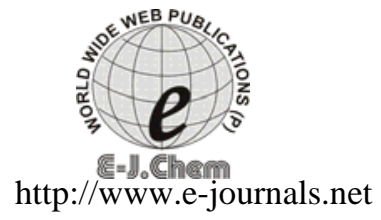

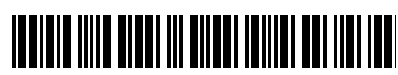

ISSN: 0973-4945; CODEN ECJHAO

E-Journal of Chemistry

2011, 8(S1), S344-S348

\title{
Corrosion Study of Iron and Copper Metals and Brass Alloy in Different Medium
}

\author{
MEMON SAMINA, ABDUL KARIM and A.VENKATACHALAM ${ }^{*}$ \\ Department of Post Graduate Studies and Research in Chemistry \\ Bhavan's College, Andheri (W), Mumbai 400058, India \\ saminamemon8@gmail.com
}

Received 30 October 2010; Revised 4 March 2011; Accepted 12 April 2011

\begin{abstract}
The corrosion of iron, copper and brass alloy has been investigated in different medium i.e. $\mathrm{HNO}_{3}, \mathrm{H}_{3} \mathrm{PO}_{4}, \mathrm{H}_{2} \mathrm{SO}_{4}, \mathrm{HCl}, \mathrm{CH}_{3} \mathrm{COOH}$. The study reveals that corrosion rate increases in the order iron $>$ copper $>$ brass and for the medium the corrosion follows the order $\mathrm{HNO}_{3}>\mathrm{H}_{3} \mathrm{PO}_{4}>\mathrm{H}_{2} \mathrm{SO}_{4}>\mathrm{HCl}>$ $\mathrm{CH}_{3} \mathrm{COOH}$. The rate of corrosion increases with increasing concentration of acid and with increase in time.
\end{abstract}

Keywords: Metal, Alloy Strips, Corrosion, Weight loss

\section{Introduction}

Corrosion $^{1-3}$ is defined as the destruction or deterioration of material because of reaction with its environment. Some insist there the definition should be restricted to metals but often the corrosion engineers must consider both metals and non-metals. Ceramics, rubber and other non-metallic materials, deterioration of paints and rubber by sunlight or chemicals, fluxing or lining of steel making furnace and attack of solid metal by another molten metal (Liquid metal Corrosion) are all considered to be corrosion. Corrosion can be fast or slow.

Corrosion of metal can be considered as extractive metallurgy. Rusting is a term reserved for steel and iron corrosion, although many other metals form their oxides when corrosion occurs. Practically all environments are corrosive to some degree. Air, moisture, fresh distilled, salt and mine waters, rural or urban and industrial atmosphere, steam and other gases such as $\mathrm{NH}_{3}, \mathrm{Cl}_{2}, \mathrm{H}_{2} \mathrm{~S}, \mathrm{SO}_{2}$, fuel gases, inorganic acids, organic acids, solvents, petroleum oils cause corrosion environment. Inorganic materials are more corrosive than the organics. High temperature and pressure involve more severe corrosion conditions. Anodizing of aluminum is another beneficial corrosion process used to obtain better and uniform appearance in addition to a protective corrosion product on the surface.

\section{Experimental}

Acids like $\mathrm{HCl}, \mathrm{H}_{2} \mathrm{SO}_{4}, \mathrm{H}_{3} \mathrm{PO}_{4}, \mathrm{HNO}_{3}$ and $\mathrm{CH}_{3} \mathrm{COOH}$ of different concentrations viz $0.1 \mathrm{~N}$, $0.5 \mathrm{~N}, 1 \mathrm{~N}, 4 \mathrm{~N}$, were prepared from commercially available (A.R) grades and were 
standardized. Similarly bases like $\mathrm{NaOH}, \mathrm{KOH}$ and $\mathrm{NH}_{4} \mathrm{OH}$ have been used in different concentrations ${ }^{4-6}$. Organic acids like benzoic acid and salicylic acid which are sparingly soluble in water their saturated solutions were used for corrosion studies. Small rectangular size metal-alloy plates of the size $8 \times 6 \times 0.3 \mathrm{~cm}$ were used and were dipped in the different solutions for different timings viz 1 to $5 \mathrm{~h}$. The weight loss in different solutions and the emf was measured for each metal by using the respective metal strips as an electrode and calomel electrode as the reference electrode. From the weight loss and the emf observed, a correlative study of the corrosion rate has been done. The summary of the results are given in Table $1-14$.

Table 1. Comparative corrosion study of metals and alloys in different medium in terms of weight loss (mg) parameter

\begin{tabular}{|c|c|c|c|c|}
\hline \multirow[t]{2}{*}{ S. No. } & \multirow[t]{2}{*}{ Medium } & \multicolumn{3}{|c|}{ Weight loss in, mg } \\
\hline & & $\mathrm{Fe}$ & $\mathrm{Cu}$ & Brass \\
\hline 1 & $4 \mathrm{~N} \mathrm{HCl}$ & 180 & 12 & 07 \\
\hline 2 & $4 \mathrm{~N} \mathrm{H}_{2} \mathrm{SO}_{4}$ & 550 & 37 & 33 \\
\hline 3 & $4 \mathrm{~N} \mathrm{HNO}_{3}$ & 3422 & 7536 & 2974 \\
\hline 4 & $4 \mathrm{~N} \mathrm{H}_{3} \mathrm{PO}_{4}$ & 824 & 54 & 51 \\
\hline 5 & $\mathrm{CH}_{3} \mathrm{COOH}$ & 52 & 08 & 13 \\
\hline 6 & $\mathrm{NaOH}$ & 18 & 21 & 12 \\
\hline 7 & $\mathrm{KOH}$ & 24 & 32 & 19 \\
\hline 8 & $\mathrm{NH}_{4} \mathrm{OH}$ & 30 & 19 & 09 \\
\hline 9 & Aniline & 10 & 05 & 10 \\
\hline 10 & Sea water & 142 & 04 & 17 \\
\hline 11 & Water & 20 & 18 & 16 \\
\hline 12 & $\mathrm{NaCl}$ & 12 & 16.6 & 9.5 \\
\hline 13 & $\mathrm{KCl}$ & 22 & 25 & 12 \\
\hline 14 & $\mathrm{CHCl}_{3}$ & 26 & 39 & 32 \\
\hline 15 & $\mathrm{~K}_{2} \mathrm{Cr}_{2} \mathrm{O}_{7}$ & 09 & 22 & 13 \\
\hline
\end{tabular}

Table 2. Comparative corrosion study of metals and Alloy in different medium in terms of emf (mV)

\begin{tabular}{|c|c|c|c|c|}
\hline \multirow[t]{2}{*}{ S.No } & \multirow[t]{2}{*}{ Medium } & \multicolumn{3}{|c|}{ EMF in $\mathrm{mV}$} \\
\hline & & $\mathrm{Fe}$ & $\mathrm{Cu}$ & Brass \\
\hline 1 & $4 \mathrm{~N} \mathrm{HCl}$ & 370 & 351 & 376 \\
\hline 2 & $4 \mathrm{~N} \mathrm{H}_{2} \mathrm{SO}_{4}$ & 437 & 52 & 102 \\
\hline 3 & $4 \mathrm{~N} \mathrm{HNO}_{3}$ & 488 & 46 & 356 \\
\hline 4 & $4 \mathrm{~N} \mathrm{H}_{3} \mathrm{PO}_{4}$ & 492 & 85 & 72 \\
\hline 5 & $4 \mathrm{~N} \mathrm{CH}_{3} \mathrm{COOH}$ & 500 & 100 & 132 \\
\hline 6 & $4 \mathrm{~N} \mathrm{NAOH}$ & 788 & 580 & 585 \\
\hline 7 & $4 \mathrm{~N} \mathrm{KOH}$ & 712 & 623 & 632 \\
\hline 8 & $4 \mathrm{~N} \mathrm{NH}_{4} \mathrm{OH}$ & 373 & 533 & 556 \\
\hline 9 & Aniline & 526 & 358 & 43 \\
\hline 10 & Sea water & 599 & 245 & 261 \\
\hline 11 & Water & 315 & 39 & 39 \\
\hline 12 & $\mathrm{NaCl}$ & 649 & 340 & 370 \\
\hline 13 & $\mathrm{KCl}$ & 624 & 325 & 303 \\
\hline 14 & $\mathrm{CHCl}_{3}$ & 518 & 173 & 129 \\
\hline 15 & $\mathrm{~K}_{2} \mathrm{Cr}_{2} \mathrm{O}_{7}$ & 537 & 103 & 185 \\
\hline
\end{tabular}

\section{Corrosion rate expression ${ }^{7-9}$}

The expression mile per year has been used as the most desirable way of expressing corrosion rate. The expression is readily calculated from the weight loss of the metal specimen during the corrosion test by the formula given below: 


$$
\text { mpy }=\frac{534 \mathrm{~W}}{\mathrm{DAT}}
$$

Where, $\mathrm{W}=$ Weight loss in $\mathrm{mg}, \mathrm{D}=$ Density of the Specimen $\left(\mathrm{g} / \mathrm{cm}^{3}\right), \mathrm{A}=$ Area of Specimen (sq. inch), $\mathrm{T}=$ Exposure time, (hours). In the present study, the corrosion rate is correlated to the weight loss in $\mathrm{mg}$.

\section{Results and Discussion}

Corrosion of iron ${ }^{10-12}$ in the form of small plate has been studied in different medium by weight loss method. Five different concentrations viz $0.1 \mathrm{~N}, 0.5 \mathrm{~N}, 1 \mathrm{~N}, 4 \mathrm{~N}$, were used for different timings viz 1-5 $\mathrm{h}$. From the study the following conclusions have been arrived.

i) The rate of corrosion increases with increasing the concentration of the acid.

ii) The rate of corrosion increases with increase in time.

The order of corrosion activity on the acids was $\mathrm{HNO}_{3}>\mathrm{H}_{3} \mathrm{PO}_{4}>\mathrm{H}_{2} \mathrm{SO}_{4}>\mathrm{HCl}>\mathrm{CH}_{3} \mathrm{COOH}>$ oxalic acid $>$ benzoic acid $>$ succinic acid $>$ salicylic acid $>\mathrm{KOH}>\mathrm{NaOH}$.

Corrosion rate on iron metal was found almost similar to acetic acid, succinic acid and salicylic acid and more at higher concentrations for oxalic and benzoic acids and it has been found to be comparatively less for bases $\mathrm{NaOH}$, and $\mathrm{KOH}$. The potential (emf) studies have also been carried out in the respective solutions on iron metal as electrode and calomel electrode as the reference electrode. For inorganic acids which show more corrosion, the emf was found to be less, and for bases, the emf was found to be more. The potential studies on Fe shows the following order bases $>$ neutral $>$ organic acids $>$ inorganic acids. This is related to the electrochemical behavior of metals under different concentrations. Similar weight loss and potential studies have also been carried out for $\mathrm{Cu}$ and brass alloy. The order of reactivity has been found to be $\mathrm{Fe}>\mathrm{Cu}>$ brass. Since brass is an alloy of $\mathrm{Cu}$ and $\mathrm{Zn}$ the coupling effect of metals do not occur and hence the corrosion rate is found to be less. This is in relation to the observed literature ${ }^{13-15}$. Solid solution alloys are usually more corrosion resistant than alloys with two or more phases.

Table 3. Weight loss of Fe in $\mathrm{HNO}_{3}$

\begin{tabular}{cccccc}
\hline Weight loss at & $1 \mathrm{~h}$ & $2 \mathrm{~h}$ & $3 \mathrm{~h}$ & $4 \mathrm{~h}$ & $5 \mathrm{~h}$ \\
\hline $0.1 \mathrm{~N} \mathrm{HNO}_{3}$ & 0.01 & 0.022 & 0.034 & 0.048 & 0.054 \\
$0.5 \mathrm{~N} \mathrm{HNO}_{3}$ & 0.11 & 0.246 & 0.427 & 0.512 & 0.556 \\
$1 \mathrm{~N} \mathrm{HNO}_{3}$ & 9.257 & 11.538 & 13.62 & 18.722 & 21.88 \\
$4 \mathrm{~N} \mathrm{HNO}_{3}$ & 12.113 & 15.246 & 19.15 & 22.52 & 34.22 \\
\hline
\end{tabular}

Table 4. Weight loss of Fe in $\mathrm{H}_{3} \mathrm{PO}_{4}$

\begin{tabular}{cccccc}
\hline Weight loss at & $1 \mathrm{~h}$ & $2 \mathrm{~h}$ & $3 \mathrm{~h}$ & $4 \mathrm{~h}$ & $5 \mathrm{~h}$ \\
\hline $0.1 \mathrm{~N} \mathrm{H}_{3} \mathrm{PO}_{4}$ & 0.028 & 0.039 & 0.042 & 0.059 & 0.078 \\
$0.5 \mathrm{~N} \mathrm{H}_{3} \mathrm{PO}_{4}$ & 0.063 & 0.236 & 0.273 & 0.349 & 0.435 \\
$1 \mathrm{~N} \mathrm{H}_{3} \mathrm{PO}_{4}$ & 0.085 & 0.325 & 0.438 & 0.664 & 0.775 \\
$4 \mathrm{~N} \mathrm{H}_{3} \mathrm{PO}_{4}$ & 0.125 & 0.439 & 0.562 & 0.688 & 0.824 \\
\hline
\end{tabular}

Table 5. Weight loss of $\mathrm{Fe}$ in $\mathrm{NaOH}$

\begin{tabular}{cccccc}
\hline Weight loss at & $1 \mathrm{~h}$ & $2 \mathrm{~h}$ & $3 \mathrm{~h}$ & $4 \mathrm{~h}$ & $5 \mathrm{~h}$ \\
\hline $0.1 \mathrm{~N} \mathrm{NaOH}$ & 0.001 & 0.002 & 0.002 & 0.003 & 0.005 \\
$0.5 \mathrm{~N} \mathrm{NaOH}$ & 0.002 & 0.002 & 0.003 & 0.004 & 0.007 \\
$1 \mathrm{~N} \mathrm{NaOH}$ & 0.001 & 0.003 & 0.004 & 0.006 & 0.008 \\
$4 \mathrm{~N} \mathrm{NaOH}$ & 0.003 & 0.006 & 0.01 & 0.012 & 0.018 \\
\hline
\end{tabular}


Table 6. Weight loss of Fe in $\mathrm{KOH}$

\begin{tabular}{cccccc}
\hline Weight loss at & $1 \mathrm{~h}$ & $2 \mathrm{~h}$ & $3 \mathrm{~h}$ & $4 \mathrm{~h}$ & $5 \mathrm{~h}$ \\
\hline $0.1 \mathrm{~N} \mathrm{KOH}$ & 0.001 & 0.002 & 0.003 & 0.004 & 0.005 \\
$0.5 \mathrm{~N} \mathrm{KOH}$ & 0.002 & 0.004 & 0.006 & 0.008 & 0.008 \\
$1 \mathrm{~N} \mathrm{KOH}$ & 0.0025 & 0.006 & 0.008 & 0.012 & 0.016 \\
$4 \mathrm{~N} \mathrm{KOH}$ & 0.005 & 0.012 & 0.018 & 0.02 & 0.024 \\
\hline
\end{tabular}

Table 7. Weight loss of $\mathrm{Cu}$ in $\mathrm{HNO}_{3}$

\begin{tabular}{cccccc}
\hline Weight loss at & $1 \mathrm{~h}$ & $2 \mathrm{~h}$ & $3 \mathrm{~h}$ & $4 \mathrm{~h}$ & $5 \mathrm{~h}$ \\
\hline $0.1 \mathrm{~N} \mathrm{HNO}_{3}$ & 0.003 & 0.004 & 0.005 & 0.006 & 0.007 \\
$0.5 \mathrm{~N} \mathrm{HNO}_{3}$ & 0.002 & 0.003 & 0.007 & 0.013 & 0.017 \\
$1 \mathrm{~N} \mathrm{HNO}_{3}$ & 0.001 & 0.002 & 0.003 & 0.004 & 0.007 \\
$4 \mathrm{~N} \mathrm{HNO}_{3}$ & 1.21 & 2.283 & 4.276 & 5.086 & 7.536 \\
\hline
\end{tabular}

Table 8. Weight loss of $\mathrm{Cu}$ in $\mathrm{H}_{3} \mathrm{PO}_{4}$

\begin{tabular}{cccccc}
\hline Weight loss at & $1 \mathrm{~h}$ & $2 \mathrm{~h}$ & $3 \mathrm{~h}$ & $4 \mathrm{~h}$ & $5 \mathrm{~h}$ \\
\hline $0.1 \mathrm{~N} \mathrm{H}_{3} \mathrm{PO}_{4}$ & 0.001 & 0.004 & 0.005 & 0.007 & 0.009 \\
$0.5 \mathrm{~N} \mathrm{H}_{3} \mathrm{PO}_{4}$ & 0.004 & 0.006 & 0.009 & 0.012 & 0.015 \\
$1 \mathrm{~N} \mathrm{H}_{3} \mathrm{PO}_{4}$ & 0.007 & 0.009 & 0.013 & 0.018 & 0.022 \\
$4 \mathrm{~N} \mathrm{H}_{3} \mathrm{PO}_{4}$ & 0.013 & 0.024 & 0.03 & 0.045 & 0.054 \\
\hline
\end{tabular}

Table 9. Weight loss of $\mathrm{Cu}$ in $\mathrm{NaOH}$

\begin{tabular}{cccccc}
\hline Weight loss at & $1 \mathrm{~h}$ & $2 \mathrm{~h}$ & $3 \mathrm{~h}$ & $4 \mathrm{~h}$ & $5 \mathrm{~h}$ \\
\hline $0.1 \mathrm{~N} \mathrm{NaOH}$ & 0.003 & 0.004 & 0.006 & 0.008 & 0.01 \\
$0.5 \mathrm{~N} \mathrm{NaOH}$ & 0.002 & 0.003 & 0.006 & 0.01 & 0.012 \\
$1 \mathrm{~N} \mathrm{NaOH}$ & 0.001 & 0.002 & 0.003 & 0.004 & 0.005 \\
$4 \mathrm{~N} \mathrm{NaOH}$ & 0.003 & 0.011 & 0.014 & 0.014 & 0.021 \\
\hline
\end{tabular}

Table10. Weight loss of $\mathrm{Cu}$ in $\mathrm{KOH}$

\begin{tabular}{cccccc}
\hline Weight loss at & $1 \mathrm{~h}$ & $2 \mathrm{~h}$ & $3 \mathrm{~h}$ & $4 \mathrm{~h}$ & $5 \mathrm{~h}$ \\
\hline $0.1 \mathrm{~N} \mathrm{KOH}$ & 0.001 & 0.002 & 0.004 & 0.006 & 0.009 \\
$0.5 \mathrm{~N} \mathrm{KOH}$ & 0.002 & 0.004 & 0.009 & 0.011 & 0.013 \\
$1 \mathrm{~N} \mathrm{KOH}$ & 0.004 & 0.016 & 0.011 & 0.013 & 0.017 \\
$4 \mathrm{~N} \mathrm{KOH}$ & 0.0075 & 0.012 & 0.019 & 0.026 & 0.032 \\
\hline
\end{tabular}

Table 11. Weight loss of Brass in $\mathrm{HNO}_{3}$

\begin{tabular}{cccccc}
\hline Weight loss at & $1 \mathrm{~h}$ & $2 \mathrm{~h}$ & $3 \mathrm{~h}$ & $4 \mathrm{~h}$ & $5 \mathrm{~h}$ \\
\hline $0.1 \mathrm{~N} \mathrm{HNO}_{3}$ & 0.002 & 0.003 & 0.004 & 0.005 & 0.006 \\
$0.5 \mathrm{~N} \mathrm{HNO}_{3}$ & 0.001 & 0.003 & 0.006 & 0.009 & 0.013 \\
$1 \mathrm{~N} \mathrm{HNO}_{3}$ & 0.001 & 0.004 & 0.006 & 0.018 & 0.019 \\
$4 \mathrm{~N} \mathrm{HNO}_{3}$ & 1.619 & 1.063 & 1.634 & 2.246 & 2.974 \\
\hline
\end{tabular}

Table 12. Weight loss of Brass in $\mathrm{H}_{3} \mathrm{PO}_{4}$

\begin{tabular}{cccccc}
\hline Weight loss at & $1 \mathrm{~h}$ & $2 \mathrm{~h}$ & $3 \mathrm{~h}$ & $4 \mathrm{~h}$ & $5 \mathrm{~h}$ \\
\hline $0.1 \mathrm{~N}$ phosphoric acid & 0.005 & 0.009 & 0.012 & 0.018 & 0.026 \\
$0.5 \mathrm{~N}$ phosphoric acid & 0.008 & 0.013 & 0.017 & 0.026 & 0.036 \\
$1 \mathrm{~N}$ phosphoric acid & 0.011 & 0.016 & 0.022 & 0.034 & 0.042 \\
$4 \mathrm{~N}$ phosphoric acid & 0.017 & 0.029 & 0.038 & 0.046 & \\
\hline
\end{tabular}


Table 13. Weight loss of Brass in $\mathrm{NaOH}$

\begin{tabular}{cccccc}
\hline Weight loss at & $1 \mathrm{~h}$ & $2 \mathrm{~h}$ & $3 \mathrm{~h}$ & $4 \mathrm{~h}$ & $5 \mathrm{~h}$ \\
\hline $0.1 \mathrm{~N} \mathrm{NaOH}$ & 0.006 & 0.007 & 0.009 & 0.096 & 0.104 \\
$0.5 \mathrm{~N} \mathrm{NaOH}$ & 0.011 & 0.01 & 0.09 & 0.007 & 0.006 \\
$1 \mathrm{~N} \mathrm{NaOH}$ & 0.017 & 0.005 & 0.003 & 0.002 & 0.001 \\
$4 \mathrm{~N} \mathrm{NaOH}$ & 0.003 & 0.007 & 0.009 & 0.0109 & 0.012 \\
\hline
\end{tabular}

Table 14.Weight loss of brass in $\mathrm{KOH}$

\begin{tabular}{cccccc}
\hline Weight loss at & $1 \mathrm{~h}$ & $2 \mathrm{~h}$ & $3 \mathrm{~h}$ & $4 \mathrm{~h}$ & $5 \mathrm{~h}$ \\
\hline $0.1 \mathrm{~N} \mathrm{KOH}$ & 0.001 & 0.002 & 0.003 & 0.005 & 0.007 \\
$0.5 \mathrm{~N} \mathrm{KOH}$ & 0.002 & 0.004 & 0.007 & 0.012 & 0.015 \\
$1 \mathrm{~N} \mathrm{KOH}$ & 0.001 & 0.003 & 0.005 & 0.008 & 0.013 \\
$4 \mathrm{~N} \mathrm{KOH}$ & 0.004 & 0.007 & 0.01 & 0.015 & 0.019 \\
\hline
\end{tabular}

\section{Conclusion}

The corrosion study results that are found out on $\mathrm{Fe}, \mathrm{Cu}$ and Brass under different media suggest that metals are highly corroded in acids, as compared to bases and neutral solutions. The rate of corrosion increases with time as well as with concentrations.

\section{References}

1. Mars G Fontana, Corrosion Engineering, $3^{\text {rd }}$ Edition, McGraw - Hill International Edition, 1987.

2. Jenny A, Charles, Griffins and Co Ltd, Anodic Oxidation of Aluminium and its Alloy, London, 1950.

3. $\quad$ Sedriks A J and Dudts P, Corrosion, 2001, 57(1), 84-91.

4. Athar M, Ali H and Quraishi M.A, Br Corrosion J., 2002, 37,155.

5. Lukovits I, Kalman E and Zucchi F, Corrosion, 2001, 57, 3.

6. McCafferty E, Corros Sci., 2003, 45, 1421-1438.

7. Ma H, Chen S, Yin B, Zhao S and Liu X, Corros Sci., 2003, 45, 867-882.

8. Bentiss F, Traisnel M and Lagrene's M, Corros Sci., 2000, 42, 127.

9. Christopher M A B adn Isabel A R B Jenny, Corros Sci, 1994, 36, 15.

10. Shastri V S and Perumareddi J R, Corrosion, 1997, 53, 617.

11. Abdul P G -Ahad and Al-Madfai S H F, Corrosion, 1989, 45,978.

12. Van Ooji W J and Zhu S, Corrosion, 2001, 57(5), 417.

13. Zaky A M and Assaf F H, Br Corros J., 2002, 37(1), 48-55.

14. Dreyman E W, ASM Hand book Corrosion, $6^{\text {th }}$ Edition, 1998, 13, 1221.

15. Mahyta A K and Al-Rawashdeh N A F, Corros Sci., 2004, 46(5), 1129-1140. 


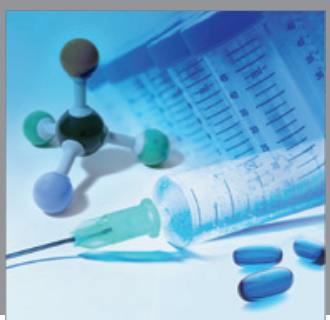

International Journal of

Medicinal Chemistry

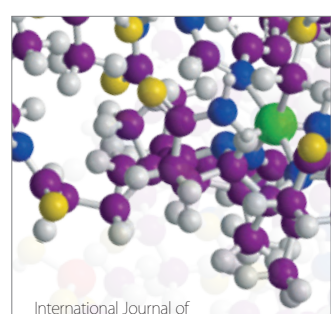

Carbohydrate Chemistry

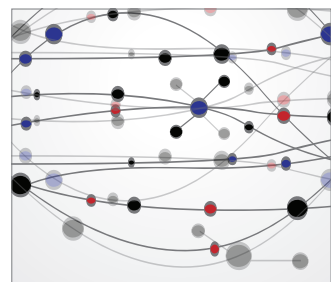

The Scientific World Journal
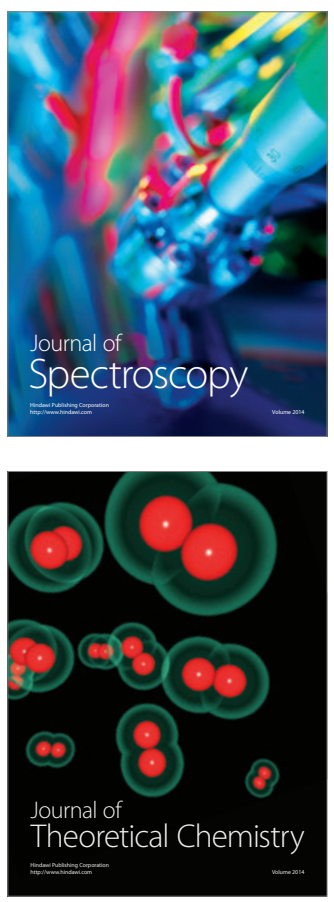
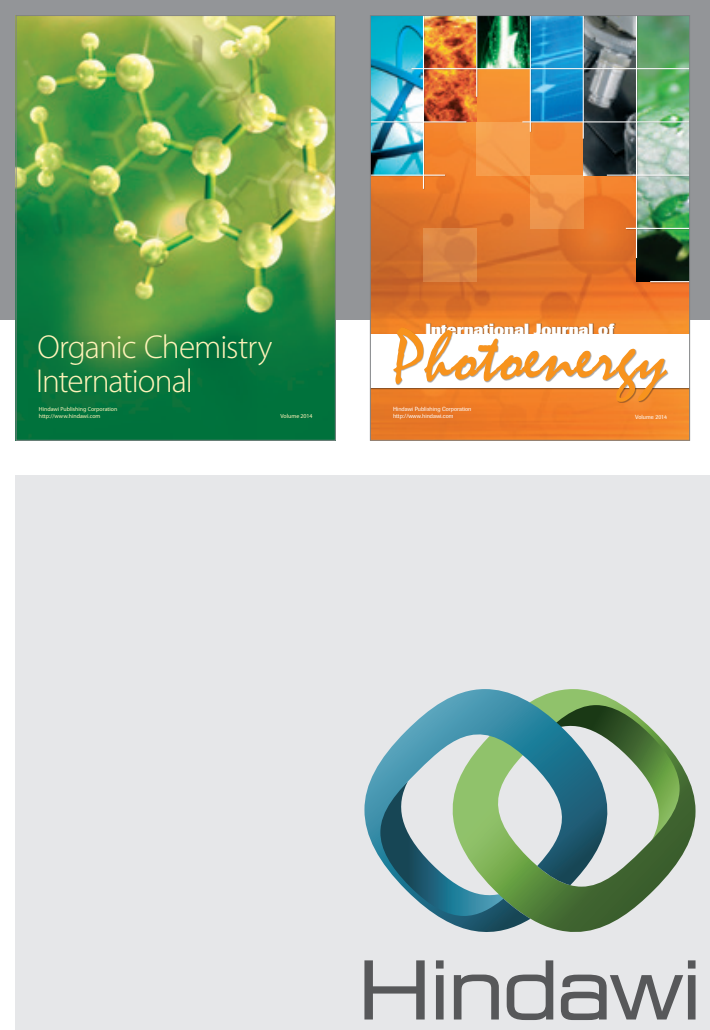

Submit your manuscripts at

http://www.hindawi.com
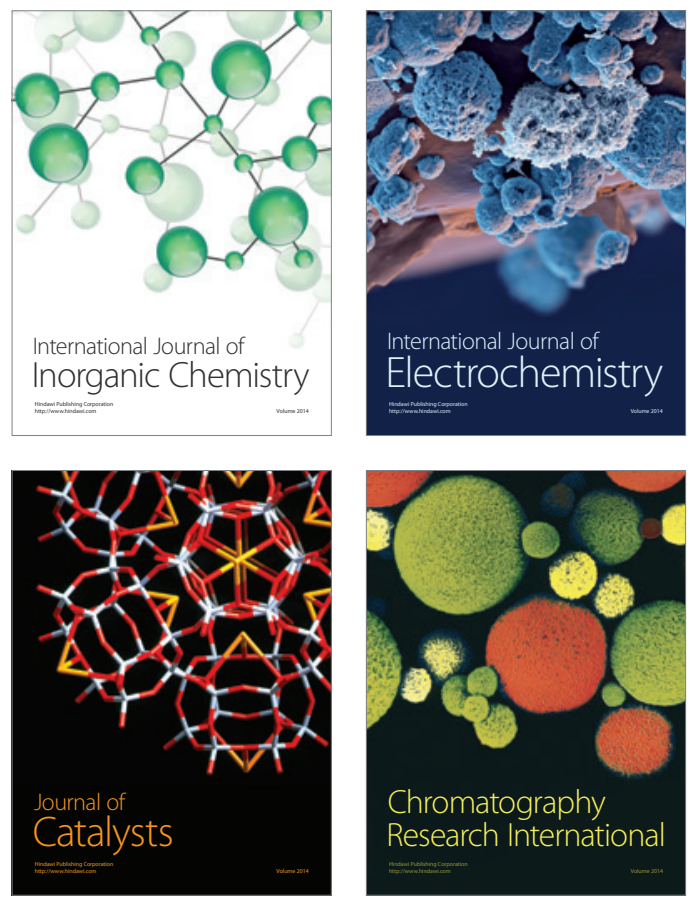
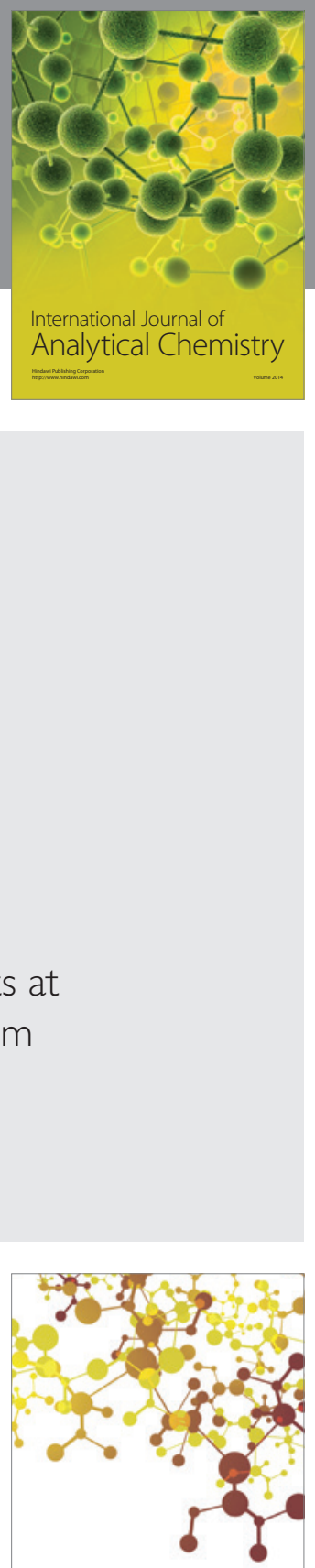

Journal of

Applied Chemistry
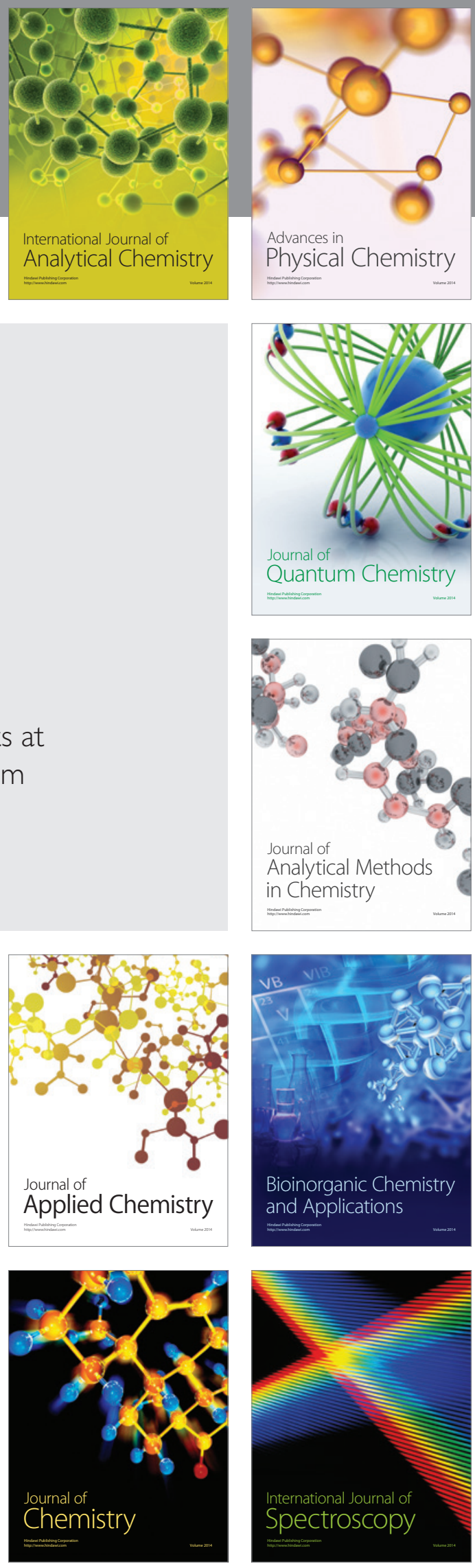